\title{
Space-Time Adaptive Processing for Distributed Aperture Radars
}

\author{
Raviraj S. Adve*, Richard A. Schneible ${ }^{\dagger}$, Michael C. Wicks ${ }^{\ddagger}$, Robert McMillan ${ }^{\S}$ \\ * Dept. of Elec. and Comp. Eng., University of Toronto, 10 King's College Road, \\ Toronto, ON M5S 3G4, Canada, rsadve@comm.utoronto.ca FAX: +1 (416) 9468765 \\ $\dagger$ Stiefvater Consultants, 10002 Hillside Terrace, Marcy, NY 13403, USA. \\ ¥ US Air Force Research Lab., Sensors Directorate, 26 Electronics Pkwy., Rome, NY 13441, USA. \\ $\S$ US Army SMDC, 106 Wynn Drive, Huntsville, AL 35805, USA.
}

Keywords: Distributed apertures, frequency diversity, range-dependent clutter

\begin{abstract}
This paper furthers the development of signal processing for distributed, waveform diverse, antenna arrays. The long term goal is to develop practical waveform-time-space adaptive processing algorithms for distributed apertures. A crucial issue identified in previous works is that, in practice, the target and interfering sources are within the near-field of the antenna array. As a first step toward the long-term goal, this paper develops the model required to generate simulated data. Such a model would be particularly useful to develop and test new adaptive signal processing algorithms. Specifically, this paper develops a model for range dependent target and interference for distributed, frequency diverse, apertures.
\end{abstract}

\section{Introduction}

In the field of radar signal processing, a recent exciting proposal has been to combine the benefits of extremely sparse arrays with the benefits of waveform diversity. Such a system is based on an array of sub-apertures placed several thousands of wavelengths apart. Waveform diversity has been proposed to deal with the resulting problems of grating lobes. Each sub-aperture of the array transmits a unique waveform, orthogonal to waveforms transmitted by the other apertures. Initial studies have shown that while providing a remarkably narrow mainbeam, such a system can also eliminate grating lobes $[1,2]$.

So far, research into waveform diverse distributed apertures has mainly been for proof-of-concept. In the area of adaptive signal processing for such systems, in particular, the studies have been limited and very preliminary $[1,3]$. The approach in these studies was to apply the existing space-time-adaptive processing
(STAP) algorithms to the waveform-time-space adaptive processing (WTSAP) case. Waveform diversity is achieved using multiple narrow band transmissions. While the results were promising, in general, the studies serve more to highlight the work remaining in developing practical adaptive processing for waveform diverse distributed apertures.

A very important result that came out of the work in $[1,2]$, is that given the extremely long baselines (thousands of wavelengths), the ranges of interest are not in the far field of the antenna array, indeed the entire notion of a steering vector has to be revisited. The range dependence of target and interference has significant impact on the performance of adaptive algorithms and requires the formulation of algorithms specifically to address this issue.

In developing adaptive signal processing for airborne radar arrays, a crucial development was the availability of data models for the target and interference [4]. This paper attempts to make a similar contribution for frequency diverse, distributed apertures, developing a model for range dependent target and interference, including frequency diversity. To account for the frequency diversity, the processing scheme uses true time delay between the widely distributed apertures. The interference is modelled as a sum of several low power interference sources, each with a range dependent contribution to the overall interference. This paper also presents numerical simulations using this model to generate data. The examples demonstrate the importance of frequency diversity in eliminating grating lobes. It is anticipated that the model will help jump start the development of WTSAP algorithms specifically for waveform diverse distributed apertures.

This paper is organized as follows: Section 2 presents the model for range dependent target and clutter data, including true time delay. Section 3 presents some numerical examples illustrating range dependence and the importance of frequency diversity in eliminating grating lobes. Finally, Section 4 draws some conclusions and points to future work. 


\section{Data Model}

In the case of airborne radar, the development of the data model depends heavily on the notion of a steering vector, the vector of signals at the ports of receiving antenna array due to a single source [4]. Because the sources are in the far-field, the steering vector, usually an array of phase shifts, depends only on the angle between the source direction and the array baseline. Adaptive processing techniques depend heavily on the availability of the target steering vector.

The situation is not as simple for distributed arrays. Given an antenna array with largest dimension $D$, operating at wavelength $\lambda$, the distance to the far field must satisfy three criteria [5]

$$
\begin{aligned}
& r \gg D, \\
& r \gg \lambda, \\
& r \gg 2 D^{2} / \lambda .
\end{aligned}
$$

Using typical values for distributed apertures, $D=$ $200 \mathrm{~m}, \lambda=0.03 \mathrm{~m}$, implies that the far field begins at a distance of approximately $2700 \mathrm{~km}$. Clearly both targets and interfering sources are not in the far field. This fact requires that any analysis of waveform diverse apertures start "from scratch". The notion of a steering vector still exists, but now depends on both angle and range, i.e., each point in space corresponds to in its own steering vector. Furthermore, coherent processing of the signals over the distributed array with frequency diversity requires true time delays, as opposed to the phase shifts used in narrowband processing. Formulating the steering vector requires accounting for all these issues.

\subsection{System Model and Steering Vector}

The abstract model of the distributed aperture using frequency diversity is as follows: The array is assumed to comprise $N$ elements distributed over the $x-y$ plane, at points $\left(x_{n}, y_{n}\right), n=1, \ldots, N$. Each element in the array transmits a coherent stream of $M$ linear-FM pulses, with common bandwidth $B$ with pulse repetition interval (PRI) $T_{r}$. However, each element transmits at a different central frequency $f_{n}, n=$ $1, \ldots N$. The transmission scheme uses true time delay to focus on a look-point $\left(X_{t}, Y_{t}, Z_{t}\right)$. This is in contrast to an airborne radar wherein a transmitting array uses phase shifts to transmit in a look direction. The return signal at all $N$ frequencies is received and processed at all $N$ elements, i.e., the return signal over space, time and frequency can be written as a length$N^{2} M$ vector.

The receiver uses true time delay to coherently process all $N$ frequencies. Denote as $D_{n}=$ $\sqrt{\left(X-x_{n}\right)^{2}+\left(Y-y_{n}\right)^{2}+\left(Z-z_{n}\right)^{2}}$, the distance of the look point to the $n^{\text {th }}$ element. The time delay used by the $n^{\text {th }}$ element on receive is

$$
\Delta T_{n}=\frac{\max \left\{D_{n}\right\}-D_{n}}{c},
$$

where $c$ is the speed of light. This is the time delay introduced to the signal at the $n^{\text {th }}$ receive element. By using true time delay, the normalized response at the $N$ elements due to all $N$ frequencies for a target at the look point is just a vector of ones, i.e., the spacetime-frequency steering vector, $\mathbf{s}$, is given by

$$
\begin{aligned}
\mathbf{s} & =\mathbf{s}_{t} \otimes \mathbf{s}_{s f}, \\
\mathbf{s}_{t} & =\left[1, e^{j 2 \pi f_{d} T_{r}}, \ldots, e^{j(M-1) \times 2 \pi f_{d} T_{r}}\right]^{T}, \\
\mathbf{s}_{s f} & =[1,1,1, \ldots, 1]^{T},
\end{aligned}
$$

where $\otimes$ denotes the Kronecker product, $f_{d}$ the target Doppler frequency, $\mathbf{s}_{t}$ the length- $M$ temporal steering vector as in [4] and $\mathbf{s}_{s f}$ the length- $N^{2}$ space-frequency steering vector of ones.

\section{$2.2 \quad$ Interference Model}

As in the case of airborne radar [4], interference here is modelled as the sum of many low power sources. However, due to frequency diversity and true time delay, interference model is far more complex than in the airborne radar case. We begin by deriving the contribution for an individual interference source for one frequency $f_{n}$. The transmitted signal over $M$ coherent pulses with pulse shape $u_{p}(t)$ is given by

$$
s(t)=u(t) e^{j 2 \pi f_{n} t+\psi} ; u(t)=\sum_{m=0}^{M-1} u_{p}\left(t-m T_{r}\right)
$$

where $\psi$ is a random phase shift. The received signal at element $i$ due to this transmitted signal at frequency $f_{n}$ is

$$
\tilde{r}_{i}^{n}(t)=A_{c} u\left(t-\tau_{i}\right) e^{j 2 \pi\left(f_{n}+f_{d c}\right)\left(t-\tau_{i}\right)},
$$

where $A_{c}$ is the complex amplitude, with random phase (also incorporating $\psi$ ), $f_{d c}$ the Doppler frequency of the interference source and $\tau_{i}=$ $\left(\sqrt{\left(x_{i}-x^{l}\right)^{2}+\left(y_{i}-y^{l}\right)^{2}+\left(z_{i}-z^{l}\right)^{2}}\right) / c$ is the delay from the $l^{\text {th }}$ interference source to the $i^{\text {th }}$ element. After down-conversion and delaying the signal by $\Delta T_{i}$, the baseband signal at element $i$ is

$$
\begin{array}{r}
r_{i}^{n}(t)=\quad A_{c} u\left(t-\tau_{i}-\Delta T_{i}\right) e^{-2 \pi f_{n}\left(\tau_{i}+\Delta T_{i}\right)} \\
\times \quad e^{j 2 \pi f_{d c} t} e^{-j 2 \pi f_{d c}\left(\tau_{i}+\Delta T_{i}\right)} .
\end{array}
$$


After matched filtering with the time reversed pulse shape, the signal becomes

$$
\begin{gathered}
x_{i}^{n}(t)=\int_{-\infty}^{\infty} r_{i}(\tau) u_{p}^{*}(\tau-t) d \tau \\
=\sum_{m=0}^{M-1} A_{c} e^{-j 2 \pi f_{n}\left(\tau_{i}+\Delta T_{i}\right)} e^{j m 2 \pi f_{d c} m T_{r}} \times \\
\int_{-\infty}^{\infty} u_{p}\left(\tau-\tau_{i}-\Delta T_{i}-m T_{r}\right) u_{p}^{*}(\tau-t) \\
e^{j 2 \pi f_{d c}\left(\tau-\tau_{i}-\Delta T_{i}-m T_{r}\right)} d \tau .
\end{gathered}
$$

The final integral is recognized as the ambiguity function of the pulse shape evaluated at the interference source Doppler $f_{d c}$. Therefore,

$$
\begin{gathered}
x_{i}^{n}(t)=\sum_{m=0}^{M-1} A_{c} e^{-j 2 \pi f_{n}\left(\tau_{i}+\Delta T_{i}\right)} e^{j 2 \pi f_{d} m T_{r}} \\
\chi\left(t-m T_{r}-\tau_{i}-\Delta T_{i}, f_{d c}\right),
\end{gathered}
$$

where $\chi(\tau, f)$ is the ambiguity function of the pulse shape $u_{p}(t)$ evaluated at delay $\tau$ and Doppler $f$. Sampling this signal every $t=k T_{s}$ corresponding to each range bin and using $\chi\left(m T_{r}, f\right) \simeq 0, m \neq 0$,

$$
\begin{aligned}
x_{i}^{n}\left(k T_{s}\right)= & \sum_{m=0}^{M-1} A_{c} e^{-j 2 \pi f_{n}\left(\tau_{i}+\Delta T_{i}\right)} e^{j 2 \pi f_{d} m T_{r}} \\
& \chi\left(k T_{s}-m T_{r}-\tau_{i}-\Delta T_{i}, f_{d c}\right),(14)
\end{aligned}
$$

Finally, given $N_{c}$ interfering sources at location $\left\{x^{l}, y^{l}, z^{l}\right\}_{l=1}^{N_{c}}$ with corresponding Doppler frequency $f_{d c}^{l}$, the received signal the $i^{\text {th }}$ element on the $m^{\text {th }}$ pulse at frequency $f_{n}$ is

$$
\begin{array}{r}
x_{i}^{n}\left(k T_{s}, m\right)=\sum_{l=1}^{N_{c}} A_{c}^{l} e^{-j 2 \pi f_{n}\left(\tau_{i}^{l}+\Delta T_{i}\right)} e^{j 2 \pi f_{n}^{l} m T_{r}} \\
\chi\left(k T_{s}-\tau_{i}^{l}-\Delta T_{i}, f_{d c}^{l}\right),
\end{array}
$$

Note that $\Delta T_{i}$, defined in Eqn. 4 , remains the delay from the look point to the $i^{\text {th }}$ element.

\section{$3 \quad$ Numerical Simulations}

This section presents the results of numerical simulations using the model developed above. In keeping with the nascent nature of this research area, the examples are preliminary in nature focusing on nonadaptive processing. They serve to illustrate the importance of frequency diversity and the need for range dependent adaptive processing. The first example illustrates the "beampattern" of the matched filter processing. Note that since the steering vector is range dependent, this is not the traditional sense of beampattern. The second example illustrates the need for frequency diversity. The first two examples do not
Table 1: Parameters common to all examples

\begin{tabular}{|c|c|c|c|}
\hline Parameter & Value & Parameter & Value \\
\hline \hline$N$ & 16 & $M$ & 8 \\
\hline$B$ & $10 \mathrm{MHz}$ & $T_{p}$ & $10 \mu s$ \\
\hline PRI & $5 T_{p}$ & Target SNR & $10 \mathrm{~dB}$ \\
\hline Target Velocity & $50 \mathrm{~m} / \mathrm{s}$ & Freq. Offset & $100 \mathrm{MHz}$ \\
\hline$X_{t}$ & $-86.51 \mathrm{~m}$ & $Y_{t}$ & $-333.12 \mathrm{~m}$ \\
\hline$Z_{t}$ & $200 \mathrm{~km}$ & INR & $50 \mathrm{~dB}$ \\
\hline
\end{tabular}

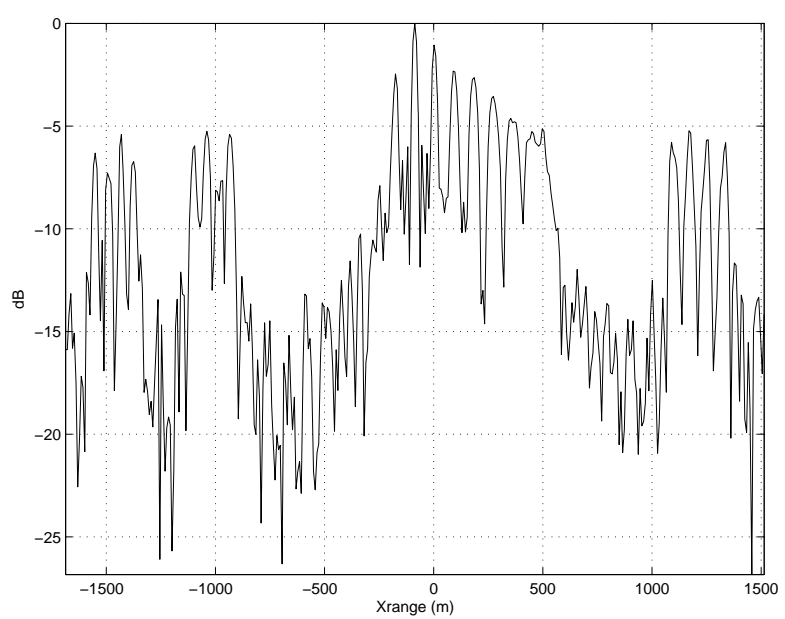

Figure 1: Matched filter processing along the transverse, $x$-direction.

include any interference. The final example includes interference and illustrates the need for adaptive processing.

All examples use the same parameters, shown in Table 1. The array uses a nominal center frequency of $10 \mathrm{GHz}$. In the table $T_{p}$ refers to the duration of each linear-FM up-chirp. The frequency offset is the difference between carrier frequencies of the $N$ transmissions. The array elements are uniformly distributed in the $x-y$ plane on a square $200 m \times 200 m$ grid. The interference-to-noise ratio (INR) is relevant only if interference data is included in the simulation.

\subsection{Example 1: Beampatterns}

The first example illustrates the transverse and "radial" beam patterns, i.e., in the $x$ - and $z$-directions. Figure 1 plots the beampattern along the $x$-direction. The figure plots the output, using matched filtering, for various values for the transverse dimension. The figure shows that the array has an approximate beamwidth of $25 \mathrm{~m}$ in the transverse direction. Note that the target is $200 \mathrm{~km}$ distant from the array in the radial, $z$-direction. 


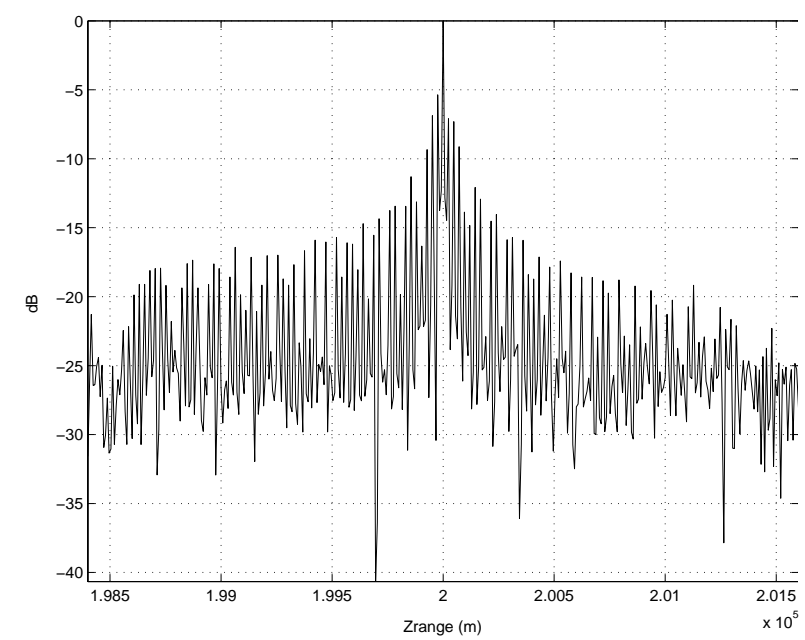

Figure 2: Matched filter processing along the radial, $z$-direction.

Figure 2 plots the beampattern along the radial $z$-direction. From the figure, the range resolution is approximately $2 \mathrm{~m}$. In both cases, the distributed aperture, coupled with frequency diversity, remarkably good resolution in both radial and transverse directions.

\subsection{Example 2: Need for Diversity}

This example illustrates the need for frequency diversity. Figure 3 plots the beampattern in the transverse, $x$-direction. Note the closely spaced grating lobes. The range dependence of the steering vector results in a very small decay in the grating lobe level further away from the target location $X_{t}$. However, clearly, the decay is inadequate for purposes of target detection. Figure 4 plots the beampattern in the radial $z$-direction. As expected, grating lobes do not occur. However, note the significantly reduced range resolution as compared to Fig. 2.

\subsection{Example 3: Including Interference}

The final example illustrates the effect of interference. Interference is modelled as a spherical cluster of $10^{4}$ low power interfering sources offset from the target location by $1.6 \mathrm{~km}$. The radius of the cluster is set to $400 \mathrm{~m}$.

Figures 5 and 6 plot the results of non-adaptive processing. Figure 5 plots the output statistic as a function of the transverse, $x$-direction, while Fig. 6 plots the output statistic as a function of the radial $z$-direction. As is clear from both figures, the strong interference completely buries the weak target.

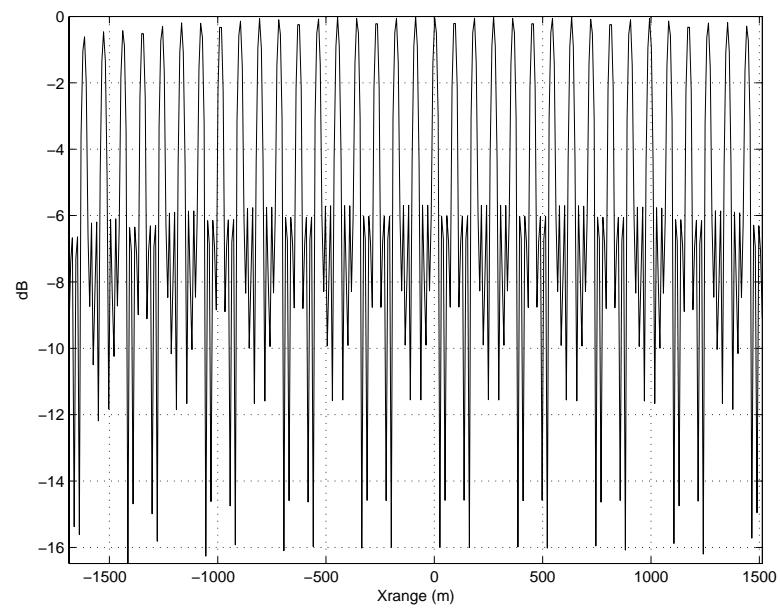

Figure 3: Matched filter processing along the transverse, $x$-direction. No frequency offset.

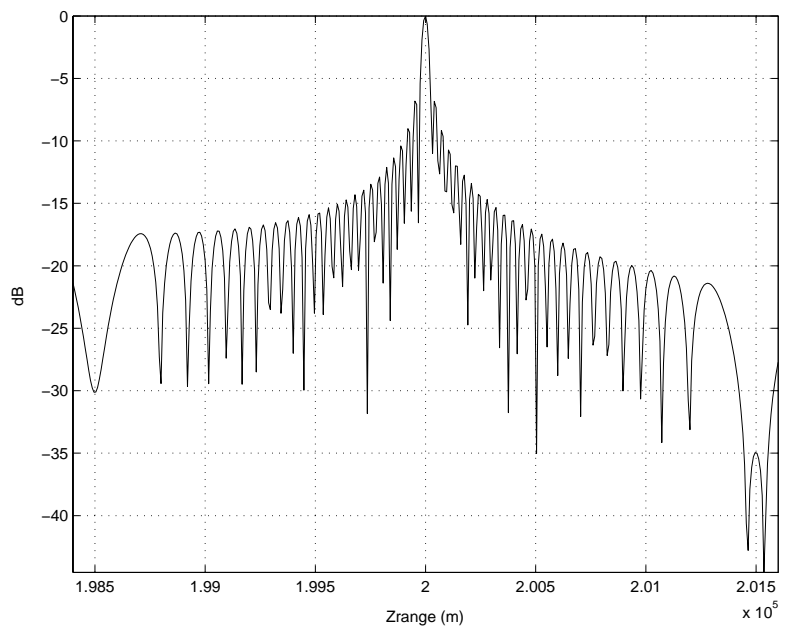

Figure 4: Matched filter processing along the radial, $z$-direction. No frequency offset.

Figure 7 plots the modified sample matrix inversion (MSMI) statistic [4] as a function of the transverse $x$-direction. This adaptive processing is implemented using only a single pulse, not the entire data cube. This is necessitated by the fact that the interference is spatially limited, i.e., the interference occupies only a few range cells, limiting the available secondary data for covariance matrix estimation. However, even with a single pulse, adaptive processing identifies the target within the strong interference.

\section{Conclusions and Future Work}

This paper has taken the initial steps toward developing adaptive processing for distributed aperture, fre- 


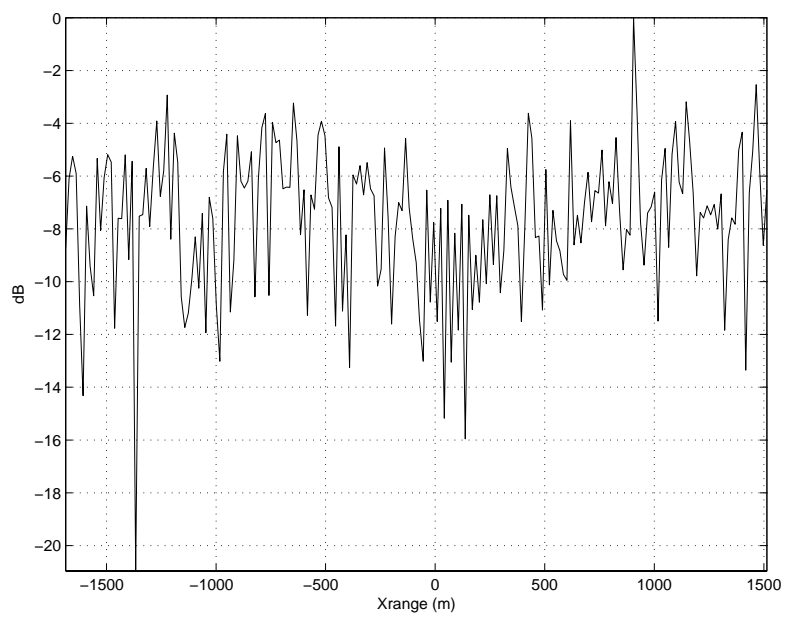

Figure 5: Matched filter processing along the transverse, $x$-direction. Includes interference.

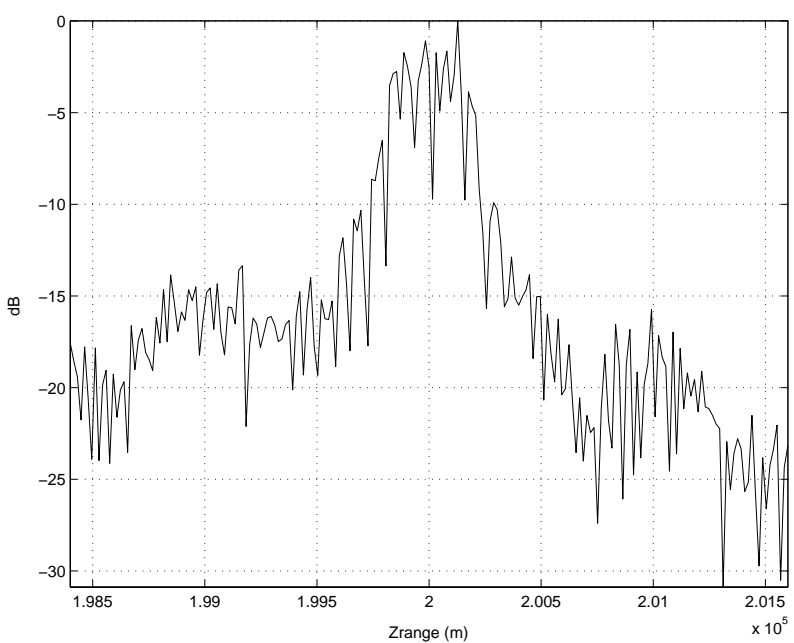

Figure 6: Matched filter processing along the radial, $z$-direction. Includes interference.

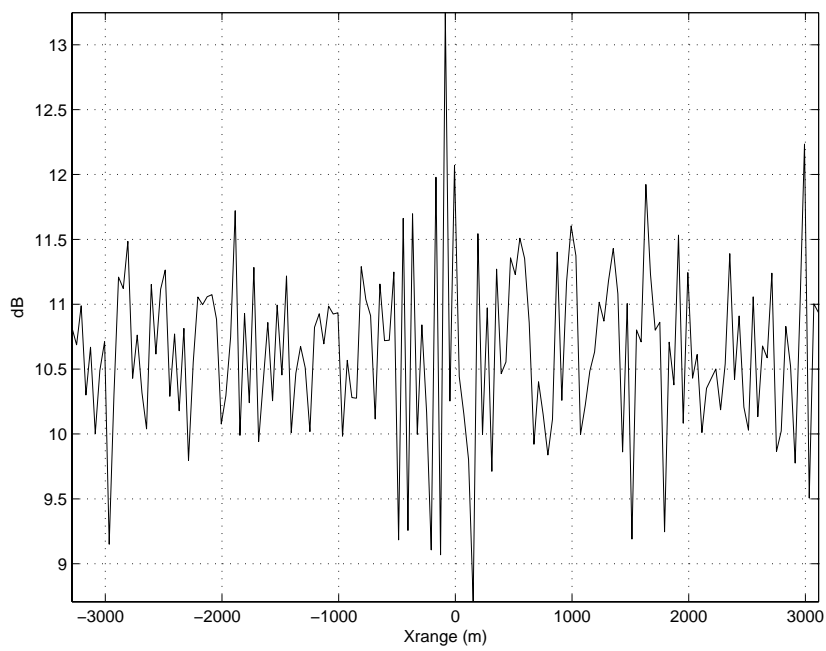

Figure 7: MSMI statistic versus transverse $x$ dimension. Includes interference. quency diverse, arrays. The steps are parallel to those undertaken in the 1990s that proved successful in the development of STAP for airborne radar, starting with the development of a data model [4]. Starting from the realization that the target and interfering source are not in the far-field of the array, this paper develops a data model accounting for range dependence while accounting for true time delay for multiple frequency bands. The numerical examples illustrate the importance of having such a data model. The data model is used here to estimate the beampattern and beamwidths in both the transverse and radial directions.

The numerical results also illustrate the crucial differences from STAP for airborne radar and the work remaining to develop a good understanding of adaptive processing for distributed apertures. As the third example shows, in crucial interference scenarios of interest, the availability of secondary data is a crucial issue. It is, therefore, likely that available adaptive algorithms, developed for airborne radar, are not relevant to the application at hand. The long-term goal of this effort is the development of adaptive algorithms specifically for distributed aperture, frequency diverse, arrays.

\section{References}

[1] R. S. Adve, "Adaptive processing for distributed aperture radars," in Proc. of the 1st Waveform Diversity Workshop, Feb. 2003. NRL, Washington, DC.

[2] R. S. Adve, R. A. Schneible, and R. McMillan, "Adaptive space/frequency processing for distributed apertures," in Proc. of 2003 IEEE Radar Conference, May 2003. Huntsville, AL.

[3] R. S. Adve, "Sub-optimal adaptive processing for distributed aperture radars," in Proc. of the 2nd Waveform Diversity Workshop, Feb. 2004. Verona, NY.

[4] J. Ward, "Space-time adaptive processing for airborne radar," Tech. Rep. F19628-95-C-0002, MIT Linclon Laboratory, December 1994.

[5] C. Balanis, Antenna Theory: Analysis and Design. John Wiley, 1997. 